\title{
COHERENT MONOIDS
}

\author{
VICTORIA GOULD
}

(Received 20 August 1990)

Communicated by P. G. Trotter

\begin{abstract}
This paper is concerned with a new notion of coherency for monoids. A monoid $S$ is right coherent if the first order theory of right $S$-sets is coherent; this is equivalent to the property that every finitely generated $S$-subset of every finitely presented right $S$-set is finitely presented. If every finitely generated right ideal of $S$ is finitely presented we say that $S$ is weakly right coherent. As for the corresponding situation for modules over a ring, we show that our notion of coherency is related to products of flat left $S$-sets, although there are some marked differences in behaviour from the case for rings. Further, we relate our work to ultraproducts of flat left $S$-sets and so to the question of axiomatisability of certain classes of left $S$-sets.

We show that a monoid $S$ is weakly right coherent if and only if the right annihilator congruence of every element is finitely generated and the intersection of any two finitely generated right ideals is finitely generated. A similar result describes right coherent monoids. We use these descriptions to recognise several classes of (weakly) right coherent monoids. In particular we show that any free monoid is weakly right (and left) coherent and any free commutative monoid is right (and left) coherent.
\end{abstract}

1991 Mathematics subject classification (Amer. Math. Soc.): 20 M 10.

\section{Introduction}

There are several obvious candidates for the definition of right coherent monoid. In [22] Wheeler defines a coherent theory (in a first order language). The theory of right $S$-sets over a monoid $S$ is coherent in Wheeler's sense if and only if every finitely generated $S$-subset of every finitely presented right $S$-set is finitely presented; we will say that such monoids are right coherent. If every finitely generated right ideal of a monoid $S$ is finitely presented we

(C) 1992 Australian Mathematical Society 0263-61 15/92 \$A2.00+0.00 
say that $S$ is weakly right coherent. These notions of coherency appeared in [12] in connection with the axiomatisability of certain classes of right $S$-sets.

Right coherent rings have been investigated by a number of authors. We recall that a ring $R$ (with unity) is right coherent if every finitely generated $R$-submodule of every finitely presented right $R$-module is finitely presented, that is, the theory of right $R$-modules is coherent in Wheeler's sense. This is equivalent $[5,8]$ to the condition that every finitely generated right ideal of $R$ is finitely presented. Chase also proved in [5] that a ring $R$ is right coherent if and only if every product of flat left $R$-modules is flat; moreover he described such rings internally as those for which the right annihilator ideals of elements are finitely generated and the intersection of any two finitely generated right ideals is finitely generated. Taking inspiration from the results of Chase, Bulman-Fleming and McDowell define a monoid to be right coherent if every product of flat left $S$-sets is flat. In Section 5 we point out some connections between our approach and theirs.

Following Section 2, where we give some preliminaries, we investigate in Section 4 some connections between noetherian properties of a monoid and coherency. If every right congruence on $S$ is finitely generated then every finitely generated right $S$-set is finitely presented, a result due to Normak [18]. Certainly then $S$ is right coherent. However it is not true that if every right ideal of $S$ is finitely generated then $S$ is weakly right coherent. But analogously to the result of Chase, one can show that a monoid $S$ is weakly right coherent if and only if the right annihilator congruence of every element is finitely generated and the intersection of any two finitely generated right ideals is finitely generated. A similar result describes right coherent monoids.

We note that any right abundant monoid has the property that the right annihilator of any element is finitely generated. This enables us to recognise immediately several examples of weakly right coherent monoids. Further, we use our description of right coherent monoids to show that, for example, semilattices of groups are right coherent.

Section 4 is concerned with the free commutative monoids $\mathscr{F} \mathscr{C}_{X}^{*}$ and the free monoid $\mathscr{F}_{X}^{*}$ on a given set $X$. Using results from Section 3 we show that $\mathscr{F} \mathscr{C}_{X}^{*}$ is right (and left) coherent and $\mathscr{F}_{X}^{*}$ is weakly right (and left) coherent.

The final section considers some relations between weak right coherency, products and ultraproducts of flat left $S$-sets, and the work of BulmanFleming and McDowell [4]. In view of Los's theorem this is also connected with the question of determining, for which monoids $S$ is the class of flat left $S$-sets (first order) axiomatisable? For completeness we also describe those monoids for which every product of strongly flat left $S$-sets is strongly flat. 


\section{Preliminaries}

We assume the reader has a basic knowledge of semigroup theory, including a familiarity with the tensor product $A \otimes B$ of a right $S$-set $A$ and a left $S$-set $B$. As a reference for the background required here we recommend $[7,13]$, the notation and terminology of which we follow as far as possible, one exception being that $S$-sets are referred to in [13] (and elsewhere) as $S$ systems. We remark that when making a definition with an obvious left-right dual, we omit for the sake of brevity the statement of the dual.

We recall from the introduction that a monoid $S$ is right coherent (weakly right coherent) if every finitely generated $S$-subset of every finitely presented right $S$-set $(S)$ is finitely presented.

Proposition 2.1 [12, Theorem 6]. A monoid $S$ is right coherent if and only if every finitely generated $S$-subset of every right $S$-set of the form $S / \rho$, where $\rho$ is a finitely generated right congruence, is finitely presented.

It is a consequence of a general result concerning finite presentations (see $[22$, p. 326]) that a monoid $S$ is right coherent (weakly right coherent) if and only if the kernel of every $S$-homomorphism $\phi: F^{n} \rightarrow S / \rho\left(\phi: F^{n} \rightarrow S\right)$ is finitely generated. Here $F^{n}$ is the free right $S$-set on $N$ generators, $n \in N$, and $\rho$ is a finitely generated right congruence on $S$. Usual notation for $F^{n}$ is $F^{n}=\dot{\bigcup}\left\{x_{i} S: 1 \leq i \leq n\right\}$, where $\dot{U}$ denotes disjoint union.

It is useful notationally to consider the categories of left (right) $S$-sets and $S$-homomorphisms: these we denote by $S$-Ens and Ens- $S$, respectively. Let $S$ be a monoid and $B \in$ Ens- $S$. Then $B$ is strongly flat if the functor $-\otimes B$ preserves equaliser and pullback diagrams in Ens- $S$. Strongly flat $S$-sets have a nice characterisation in terms of elements.

Proposition 2.2 [21]. A left $S$-set $B$ is strongly flat if and only if for all $s, t \in S$ and $a, b \in B$, if $s a=t b$ then there are $s^{\prime}, t^{\prime} \in S$ and $c \in B$ with $s s^{\prime}=t t^{\prime}, a=s^{\prime} c$ and $b=t^{\prime} c$; moreover if $a=b$ then we can take $s^{\prime}=t^{\prime}$.

Note that in [21], strong flatness is referred to as weak flatness. The notion of strongly flat is rather restrictive in the sense that all left $S$-sets are strongly flat if and only if $S$ is trivial [16].

The left $S$-set $B$ is $f$ lat if the functor $-\otimes B$ preserves monomorphisms (which are here injections) in Ens- $S$. If $-\otimes B$ preserves embeddings of right ideals of $S$ into $S$ (considered as a right $S$-set), then $B$ is called weakly flat. Since monomorphisms are equalisers in Ens- $S$, every strongly flat left $S$-set is flat; clearly every flat left $S$-set is weakly flat. 
A monoid $S$ is left absolutely flat if every left $S$-set is flat. Such monoids have been wisely investigated, in particular by S. Bulman-Fleming and K. McDowell. They form an extensive class-for example it is shown in [1] that every inverse monoid is left and right absolutely flat. Thus every non-trivial inverse monoid $S$ has a left $S$-set which is flat but not strongly flat. Further, by the results of [2] and [3], given any right normal band $S$ which does not have constant structure mappings then there is a weakly flat $S^{1}$-set which is not flat.

For $A \in$ Ens- $S$ and $a, b \in A$ we define

$$
\begin{aligned}
R(a, b) & =\{(u, v) \in S \times S: a u=b v\}, \\
r(a, b) & =\{u \in S: a u=b u\}, \quad r(a)=R(a, a) .
\end{aligned}
$$

Then $R(a, b)=\varnothing$ or is an $S$-subset of the right $S$-set $S \times S, r(a, b)=\varnothing$ or is a right ideal of $S$ and $r(a)$ is a right congruence on $S$ the right annihilator congruence of $a$.

If $A \in$ Ens- $S$ and $H \subseteq A \times A$ then we denote by $\rho(H)$ the smallest congruence on $A$ containing $H$. As a special case of the Mal'cev Lemma we have the following.

Lemma 2.3. Given $a \in$ Ens- $S, H \subseteq A \times A$ and $a, b \in A$, then $a \rho(H) b$ if and only if $a=b$ or there is a sequence

$$
a=c_{1} t_{1}, d_{1} t_{1}=c_{2} t_{2}, \ldots, d_{l-1} t_{l-1}=c_{l} t_{l}, d_{l} t_{l}=b,
$$

where for $i \in\{1, \ldots, l\}, t_{i} \in S$ and $\left(c_{i}, d_{i}\right) \in H \cup H^{-1}$.

A sequence as in Lemma 2.3 will be referred to as a $\rho(H)$-sequence of length $n$. For any $A \in$ Ens- $S$ and congruence $\rho$ on $A$, we denote by [a] the $\rho$-equivalence class of an element $a$ of $A$.

Finally in this section we quote a result from [1] which determines when two elements in a tensor product of $S$-sets are equal.

Lemma 2.4. Let $S$ be a monoid, $A \in$ Ens- $S, B \in S$-Ens. Then $a \otimes b=$ $a^{\prime} \otimes b^{\prime}$ in $A \otimes B$ if and only if there exist $a_{1}, \ldots, a_{n} \in A, b_{2}, \ldots, b_{n} \in B$, $s_{1}, \ldots s_{n}, t_{1}, \ldots, t_{n} \in S$ such that

$$
\begin{array}{rlrl}
a= & a_{1} s_{1}, & \\
a_{1} t_{2}= & a_{2} s_{2}, & s_{1} b=t_{1} b_{2}, \\
a_{2} t_{2}=a_{3} s_{3}, & s_{2} b_{2}=t_{2} b_{3}, \\
\cdot & \cdot \\
a_{n} t_{n}=a^{\prime}, & s_{n} b_{n}=t_{n} b^{\prime} .
\end{array}
$$


A system of equalities as in Lemma 2.4 is called a scheme over $A$ and $B$ of length $n$.

\section{Noetherian conditions and coherency}

If a ring $R$ is right noetherian, that is, every right ideal is finitely generated, then it is well known (see, for example [20]) that every finitely generated right $R$-module is finitely presented, so that certainly $R$ is right coherent. Of course for rings a right congruence is determined by a right ideal, which is not true for monoids in general. We shall say that a monoid $S$ is right noetherian (weakly right noetherian) if every right congruence on $S$ (right ideal of $S$ ) is finitely generated. By considering Rees right congruences it is easy to see that every right noetherian monoid is weakly right noetherian.

Thus two questions naturally arise. First, is every right noetherian monoid right coherent? And second, is every weakly right noetherian monoid weakly right coherent? The first is answered in the affirmative by Normak, who shows in [18] that every finitely generated right $S$-set over a right noetherian monoid $S$ is finitely presented. The second is answered negatively by the following example, for which the author is grateful to J. B. Fountain.

EXAMPLE 3.1. Let $G$ be an abelian group which is not finitely generated. Let $x, 0$ be symbols not in $G$. Put $S_{0}=G, S_{1}=\{x g: g \in G\}, S_{2}=$ $\left\{x^{2} g: g \in G\right\}, S_{3}=\left\{x^{3}\right\}, S_{4}=\{0\}$ (here $x^{2}, x^{3}$ are merely symbols). Put $S=\bigcup\left\{S_{i}: 0 \leq i \leq 4\right\}$ and define a multiplication on $S$ extending that of $G=S_{0}$ as follows:

$$
\begin{aligned}
& g(x h)=x(g h)=(x h) g, \\
& g\left(x^{2} h\right)=x^{2}(g h)=\left(x^{2} h\right) g, \\
& g x^{3}=x^{3}=x^{3} g, \\
& (x g)(x h)=x^{2}(g h)=(x h)(x g), \\
& (x g)\left(x^{2} h\right)=x^{3}=\left(x^{2} h\right)(x g), \\
& (x g) x^{3}=0=x^{3}(x g), \\
& \left(x^{2} g\right)\left(x^{2} h\right)=0=\left(x^{2} h\right)\left(x^{2} g\right), \\
& \left(x^{2} g\right) x^{3}=0=x^{3}\left(x^{2} g\right), \\
& x^{3} x^{3}=0=0 s=s 0 \text { for all } s \in S .
\end{aligned}
$$

For ease of notation we identify $x, x^{2}$ with $x 1_{G}, x^{2} 1_{G}$ respectively. It is not difficult to see that $S$ is a monoid which is weakly right noetherian. Indeed, every right ideal is principal. However, we claim that the right ideal $x^{2} S$ is not finitely presented so that $S$ is not weakly right coherent. Since $x^{2} S \cong S / r\left(x^{2}\right)$, by remarks in the previous section it is enough to show that $r\left(x^{2}\right)$ is not finitely generated. 
Suppose therefore that $k$ is a natural number and

$$
H=\left\{\left(\alpha_{1}, \beta_{1}\right), \ldots,\left(\alpha_{k}, \beta_{k}\right)\right\}
$$

is a symmetric set of generators for $r\left(x^{2}\right)$. Let $g \in G$, where $g \neq 1_{G}$. Then $(x g, x) \in r\left(x^{2}\right)$ and as $x g \neq x, x g$ and $x$ are connected by a $\rho(H)$-sequence

$$
x g=c_{1} t_{1}, d_{1} t_{1}=c_{2} t_{2}, \ldots, d_{n} t_{n}=x
$$

which we may assume is of minimal length. From the construction of $S$ it follows that each pair $\left(c_{i}, d_{i}\right)$ is of the form $\left(x h_{i}, x k_{i}\right)$ where $h_{i}, k_{i} \in G$ and each $t_{i} \in G, 1 \leq i \leq n$. One thus obtains that $g=h_{1} k_{1}^{-1} \cdots h_{n} k_{n}^{-1}$. It is now an easy consequence that $G$ is finitely generated. From this contradiction we have that $S$ is not weakly right coherent.

On the positive side we have the following.

Proposition 3.2. Let $A$ be a right $S$-set. Then the following conditions are equivalent:

(i) the kernel of every $S$-homomorphism from a finitely generated free right $S$-set to $A$ is finitely generated;

(ii) for all $a, b \in A, r(a)$ is finitely generated and $a S \cap b S=\varnothing$ or is finitely generated.

Proof. (i) $\Rightarrow$ (ii) Let $a, b \in A$. Define $\phi: S \rightarrow A$ by $s \phi=a s$. Then $\operatorname{ker} \phi=r(a)$ and is finite generated by assumption.

Consider now $\psi: F=x S \cup y S \rightarrow A$ where $x \psi=a$ and $y \psi=b$. Again by assumption, $\operatorname{ker} \psi=\langle H\rangle$ for some finite subset $H$ of $F \times F$, where we may suppose that $H$ is symmetric. Let

$$
C=\bigcup\{a s S:(x s, y t) \in H \text { for some } t \in S\} ;
$$

clearly $C$ is empty or is a finitely generated $S$-subset of $A$. We claim that $C=a S \cap b S$.

Given $(x s, y t) \in H$ then $a s=b t \in a S \cap b S$ so that $a s S \subseteq a S \cap b S$. Thus $C \subseteq a S \cap b S$. Conversely, suppose that $a u=b v \in a S \cap b S$, where $u, v \in S$. Then $(x u, y v) \in \operatorname{ker} \psi$ and as $x u \neq y v$ there is a $\rho(H)$-sequence

$$
x u=c_{1} t_{1}, d_{1} t_{1}=c_{2} t_{2}, \ldots, d_{l} t_{l}=y v .
$$

Let $n$ be the least number such that $\left(c_{n}, d_{n}\right)=\left(x h_{n}, y k_{n}\right)$. Then for $1 \leq$ $i<n,\left(c_{i}, d_{i}\right)=\left(x h_{i}, x k_{i}\right)$ and

$$
a u=a h_{1} t_{1}=a k_{1} t_{1}=a h_{2} t_{2}=\cdots=a h_{n} t_{n}
$$

so that $a u \in C$. It follows that $a S \cap b S=C$ as required. 
(ii) $\Rightarrow$ (i) Let $\phi: x_{1} S \cup \cdots \cup x_{n} S \rightarrow A$ where $x_{i} \phi=a_{i}$. For $i \in\{1, \ldots, n\}$ we have that $x_{i} s \operatorname{ker} \phi x_{i} t$ if and only if $a_{i} s=a_{i} t$ if and only if $(s, t) \in$ $r\left(a_{i}\right)$ and by assumption $r\left(a_{i}\right)$ has a finite set of generators $H_{i}$. Thus $x_{i} H_{i}:=\left\{\left(x_{i} h, x_{i} k\right):(h, k) \in H_{i}\right\} \subseteq \operatorname{ker} \phi$ and $x_{i} s \operatorname{ker} \phi x_{i} t$ if and only if $x_{i} s\left\langle x_{i} H_{i}\right\rangle x_{i} t$.

For $i, j \in\{1, \ldots, n\}$ where $i \neq j$, let $C_{i j}=a_{i} S \cap a_{j} S$. If $C_{i j} \neq \varnothing$ then $C_{i j}=\bigcup\left\{b_{i j}^{k} S: 1 \leq k \leq p(i j)\right\}$ for some $p(i j) \in N$ and for each $k \in\{1, \ldots, p(i j)\}, b_{i j}^{k}=a_{i} u_{i j}^{k}=a_{j} v_{i j}^{k}$. Let $K_{i j}=\left\{\left(x_{i} u_{i j}^{k}, x_{j} v_{i j}^{k}\right): 1 \leq\right.$ $k \leq p(i j)\}$, with the convention that if $C_{i j}=\varnothing$ then $K_{i j}=\varnothing$. Certainly $\nu=\left\langle\bigcup\left\{x_{i} H_{i}: 1 \leq i \leq n\right\} \cup \bigcup\left\{K_{i j}: 1 \leq i, j \leq n, i \neq j\right\}\right\rangle \subseteq \operatorname{ker} \phi$.

Conversely, suppose that $\left(x_{i} s, x_{i} t\right) \in \operatorname{ker} \phi$ for some $i \in\{1, \ldots, n\}$. As remarked above, $x_{i} s\left\langle x_{i} H_{i}\right\rangle x_{i} t$ so that certainly $x_{i} s \nu x_{i} t$. If $\left(x_{i} s, x_{j} t\right) \in \operatorname{ker} \phi$ where $i$ and $j$ are distinct elements of $\{1, \ldots, n\}$, then $a_{i} s=a_{j} t \in C_{i j}$ so that

$$
a_{i} s=a_{j} t=a_{i} u_{i j}^{k} r=a_{j} v_{i j}^{k} r
$$

for some $r \in S$. But then $\left(x_{i} s, x_{i} u_{i j}^{k} r\right),\left(x_{j} t, x_{j} v_{i j}^{k} r\right) \in \operatorname{ker} \phi$ so that

$$
x_{i} s \nu x_{i} u_{i j}^{k} r \nu x_{j} v_{i j}^{k} r \nu x_{j} t \text {. }
$$

Hence $\nu=\operatorname{ker} \phi$ and $\operatorname{ker} \phi$ is finitely generated.

COROLLARY 3.3. The following are equivalent for a monoid $S$ :

(i) $S$ is weakly right coherent;

(ii) for all $a, b \in S, r(a)$ is finitely generated and $a S \cap b S=\varnothing$ or is $a$ finitely generated right ideal;

(iii) for all $a, b \in S, r(a)$ is finitely generated and the intersection of any two finitely generated right ideals of $S$ is empty or is finitely generated.

COROLlaRY 3.4. The following are equivalent for a monoid $S$ :

(i) $S$ is right coherent;

(ii) for any finitely generated right congruence $\rho$ on $S$ and any $a, b \in S / \rho$, $r(a)$ is finitely generated and $a S \cap b S=\varnothing$ or is finitely generated;

(iii) for any finitely generated right congruence $\rho$ on $S$ then for any $a \in S / \rho, r(a)$ is finitely generated and the intersection of any two finitely generated $S$-subsets of $S / \rho$ is empty or is finitely generated.

Corollary 3.3 provides us with many examples of weakly right coherent monoids. We recall that the relation $\mathscr{L}^{*}$ is defined on a monoid $S$ by the rule that $a \mathscr{L}^{*} b$ if for all $x, y \in S$,

$$
a x=a y \text { if and only if } b x=b y .
$$


A monoid is right abundant if every $\mathscr{L}^{*}$-class contains an idempotent. Such monoids are also called right principally projective or right $\mathrm{PP}$, since they are precisely those monoids in which every principal right ideal is projective. These monoids were first considered in [14], and have subsequently been studied by Fountain in $[9,10]$. They also occur in the work of BulmanFleming and McDowell (see [3] and [4]). If $S$ is a regular monoid then $\mathscr{L}^{*}=\mathscr{L}$ so that certainly $S$ is right abundant. Obviously left cancellative monoids are also right abundant.

LEMMA 3.5. If $S$ is right abundant then $r(s)$ is finitely generated for all $s \in S$.

Proof. If $S$ is right abundant and $s \in S$ then $r(s)=\langle(1, e)\rangle$ where $s \mathscr{L}^{*} e$ and $e \in E(S)$.

Corollary 3.6. Let $S$ be a monoid. Any of the following conditions is sufficient for $S$ to be weakly right coherent:

(i) $S$ is right abundant and for all $a, b \in S$, if $a S \cap b S \neq \varnothing$ then $a S \cap b S$ is finitely generated;

(ii) $S$ is right abundant and weakly right noetherian;

(iii) $S=T^{1}$ where $T$ is completely (0-) simple;

(iv) $S$ is inverse;

(v) $S$ is the multiplicative semigroup of a regular ring.

Proof. Everything is clear except perhaps (v). The fact that the intersection of two principal right ideals in a regular ring is again principal is well known. For a reference, we quote [17] together with [5]. On p. 68 the former states that in a regular ring, every finitely generated right ideal is principal. Thus if $I$ is a finitely generated right ideal of a regular ring $R$, then $I=a R$ for some element $a$ in $R$. As $r(a)$ is finitely generated it follows that $I$ is finitely presented ("finitely related" in the terminology of [5]). Then Theorem 2.2 of [5] gives that the intersection of two principal right ideals is finitely generated and hence principal.

We remark that in view of the comments following Proposition 5.4, parts (iii), (iv) and (v) of Corollary 3.6 also follow from [4, Theorem 7].

Our next aim is to use Corollary 3.4 to obtain examples of right coherent monoids.

LEMma 3.7. Let $S$ be a monoid such that given any $s \in S$ and finite subset $X$ of $S$, there is an $e \in E(S)$ such that sRe and $e$ commutes with 
all $x \in X$. Then for every finitely generated right congruence $\rho$ on $S$ and every $a \in S / \rho, r(a)$ is finitely generated.

Proof. Let $\rho$ be finitely generated by $H=\left\{\left(u_{1}, v_{1}\right), \ldots,\left(u_{n}, v_{n}\right)\right\}$. Put $X=\left\{u_{1}, v_{1}, u_{2}, \ldots, v_{n}\right\}$ and suppose that $a=[s] \in S / \rho$. By hypothesis we may choose $e \in E(S)$ with $s \mathscr{R} e$ such that $e$ commutes with every element of $X$. Pick $s^{\prime} \in S$ such that $s=s s^{\prime} s$ and $e=s s^{\prime}$, so that $\left(1, s^{\prime} s\right) \in$ $r(a)$. Further, for any $i \in\{1, \ldots, n\}, s s^{\prime} u_{i}=u_{i} s s^{\prime} \rho v_{i} s s^{\prime}=s s^{\prime} v_{i}$, so that $\left(s^{\prime} u_{i}, s^{\prime} v_{i}\right) \in r(a)$. Thus if $\nu=\left\langle\left\{\left(1, s^{\prime} s\right),\left(s^{\prime} u_{i}, s^{\prime} v_{i}\right): 1 \leq i \leq n\right\}\right\rangle$ then $\nu \subseteq r(a)$.

Conversely, if $(p, q) \in r(a)$, then either $s p=s q$ so that $p \nu s^{\prime} s p=s^{\prime} s q \nu q$, or there is a $\rho(H)$-sequence

$$
s p=c_{1} t_{1}, d_{1} t_{1}=c_{2} t_{2}, \ldots, d_{l} t_{l}=s q .
$$

In the latter case $p \nu s^{\prime} s p=s^{\prime} c_{1} t_{1} \nu s^{\prime} d_{1} t_{1} \cdots \nu s^{\prime} d_{l} t_{l}=s^{\prime} s q \nu q$. Thus $r(a)=\nu$ and so is finitely generated.

COROLLARY 3.8. Every inverse monoid with central idempotents (right) coherent.

Proof. In view of Corollary 3.4 and Lemma 3.7 it is enough to show that for any finitely generated right congruence $\rho$ of $S$ and $a, b \in S / \rho, a S \cap b S$ is finitely generated.

Given $\rho, a, b$ as above, we suppose that $a=[s]$ and $b=[t]$. Let $s^{\prime}, t^{\prime}$ be the inverses of $s, t$ respectively. We claim that $a S \cap b S=c S$ where $c=\left[s^{\prime} s t^{\prime} t\right]$. For from the fact that $S$ is a semilattice of groups, $c=\left[s^{\prime} s t^{\prime} t\right]=\left[s s^{\prime} t^{\prime} t\right]=[s] s^{\prime} t^{\prime} t=a s^{\prime} t^{\prime} t$ and similarly, $c=b t^{\prime} s^{\prime} s$, so that $c \in a S \cap b S$ and $c S \subseteq a S \cap b S$.

Conversely, if $d=a h=b k$, then

$$
d=[s] h=[t] k=\left[t t^{\prime} t\right] k=[t] k t^{\prime} t
$$

so that

$$
d=[s] h t^{\prime} t=\left[s s^{\prime} s\right] h t^{\prime} t=\left[s^{\prime} s t^{\prime} t\right] s h=c s h \in c S .
$$

Thus the claim and hence the lemma hold.

\section{Free monoids and coherency}

For a given non-empty set $X$ we denote by $\mathscr{F}_{X},\left(\mathscr{F} \mathscr{C}_{X}\right)$ the free semigroup (free commutative semigroup) on $X$. The free monoid (free commutative monoid) on $X$ we write as $\mathscr{F}_{X}^{*}\left(\mathscr{F} \mathscr{C}_{X}^{*}\right)$. If $w \in \mathscr{F}_{X}^{*}$ or $\mathscr{F} \mathscr{C}_{X}^{*}$ the content of $w, c(w)$, is defined by $c(w)=\{x \in X: x$ occurs in $w\}$. 
Proposition 4.1. The free monoid on any non-empty set is weakly right coherent.

Proof. Let $S=\mathscr{F}_{X}^{*}$ where $X \neq \varnothing$. Since $S$ is cancellative, $S$ is certainly right abundant. It is clear that the intersection of any two principal right ideals is empty or principal, and so by Corollary $3.3, S$ is weakly right coherent.

The proof of the next lemma is routine and so is omitted. $T^{1}$.

LemMA 4.2. Let $T$ be a semigroup. If $T$ is right noetherian, then so is

Redei's Theorem [7, 19] states that $\mathscr{F} \mathscr{C}_{X}$ is (right) noetherian for any finite, non-empty set $X$. Thus in this case, certainly $\mathscr{F} \mathscr{C}_{X}^{*}$ is (right) coherent. We make use of Redei's Theorem to show that for any non-empty set $X, \mathscr{F} \mathscr{C}_{X}^{*}$ is (right) coherent.

THEOREM 4.3. For any non-empty set $X, S=\mathscr{F} \mathscr{C}_{X}^{*}$ is a right (and left) coherent monoid.

Proof. We show that conditions (ii) of Corollary 3.4 hold. Our convention is that $\mathscr{F} \mathscr{C}_{\mathscr{\varnothing}}^{*}$ is the trivial monoid. Let $H$ be a finite subset of $S \times S$ and put $\rho=\rho(H)$. Let $K$ by any finite subset of $X$ containing $U$, where $U$ consists of all letters occurring in any $u$ where $(u, v) \in H \cup H^{-1}$ for some $v \in S$.

We show that if $(s, t) \in S$ and $s \rho t$, then $(s, t)=(x, y) z$ for some $x, y \in \mathscr{F} \mathscr{C}_{K}^{*}$ and $z \in \mathscr{F} \mathscr{C}_{X \backslash K}^{*}$ such that $x \rho_{K}(H) y$ where $\rho_{K}(H)$ is the congruence on $\mathscr{F} \mathscr{C}_{K}^{*}$ generated by $H$. For if $s \rho t$, then either $s=t$ so that $(s, t)=(x, x) z$ where $x \in \mathscr{F} \mathscr{C}_{K}^{*}, z \in \mathscr{F} \mathscr{C}_{X \backslash K}^{*}$, or there is a $\rho(H)$ sequence

$$
s=c_{1} t_{1}, d_{1} t_{1}=c_{2} t_{2}, \ldots, d_{l} t_{l}=t .
$$

Now writing $s=x z$, where $c(x) \subseteq K, c(z) \subseteq X \backslash K$, we must have for each $i \in\{1, \ldots, l\}$ that $t_{i}=t_{i}^{\prime} z, t_{i}^{\prime} \in S$, and so $t_{i}^{\prime} \in \mathscr{F} \mathscr{C}_{K}^{*}, i \in\{1, \ldots, l\}$. Thus $t=y z$ where $y \in \mathscr{F} \mathscr{C}_{K}^{*}, x \rho_{K}(H) y$ and $(s, t)=(x, y) z$ as required.

Let $a \in S$ and define $K_{1}$ to be the subset of $X$ consisting of $U$ together with all letters occurring in $a$. Then $r([a]) \cap \mathscr{F} \mathscr{C}_{K_{1}}^{* 2}$ is a (right) congruence on $\mathscr{F} \mathscr{C}_{K_{1}}^{*}$ and so by Redei's theorem has a finite set $M$ of generators as a congruence on $\mathscr{F} \mathscr{C}_{K_{1}}^{*}$. But if $(s, t) \in r([a])$ then as pat so that $(a s, a t)=$ $(u, v) w$ for some $(u, v) \in \rho_{K_{1}}(H)$ and $w \in \mathscr{F} \mathscr{C}_{X \backslash K_{1}}^{*}$. Since $c(a) \subseteq K_{1}$ we 
must have that $u=a u^{\prime}, v=a v^{\prime}$ for some $u^{\prime}, v^{\prime} \in \mathscr{F} \mathscr{C}_{K_{1}}^{*}$ and so $(s, t)=$ $\left(u^{\prime}, v^{\prime}\right) w$. Further, $a u^{\prime}=u \rho v=a v^{\prime}$ so that $\left(u^{\prime}, v^{\prime}\right) \in r([a]) \cap \mathscr{F} \mathscr{C}_{K_{1}}^{* 2}$. It follows that $M$ generates $r([a])$ as a congruence on $S$.

Consider now elements $a, b \in S$ where $I=[a] S \cap[b] S \neq \varnothing$. Let $K_{2}$ be the subset of $X$ consisting of $U$ together with all letters occurring in $a$ or $b$. Since $I \neq \varnothing$ there are elements $u, v \in S$ with aupbv. But then $(a u, b v)=(x, y) z$ for some $x, y \in \mathscr{F} \mathscr{C}_{K_{2}}^{*}, z \in \mathscr{F} \mathscr{C}_{X \backslash K_{2}}^{*}$ such that $x \rho_{K_{2}}(H) y$. Thus $x=a x^{\prime}, y=b y^{\prime}$ for some $x^{\prime}, y^{\prime} \in \mathscr{F} \mathscr{C}_{K_{2}}^{*}$ and $[x]^{\prime} \in$ $J:=[a]^{\prime} S^{\prime} \cap[b]^{\prime} S^{\prime}$, where for $w \in S^{\prime}:=\mathscr{F} \mathscr{C}_{K_{2}}^{*},[w]^{\prime}$ denotes the $\rho_{K_{2}}(H)$ equivalence class of $w$. In particular, $J \neq \varnothing$ and as $S^{\prime}$ is certainly right coherent, $J$ is finitely generated as an $S^{\prime}$-subset of $S^{\prime} / \rho_{K_{2}}(H)$. It is easy to see that if $N^{\prime}=\left\{\left[r_{i}\right]^{\prime}: 1 \leq i \leq n\right\}$ generates $J$, then $N=\left\{\left[r_{i}\right]: 1 \leq i \leq n\right\}$ generates $I$, thus completing the proof.

\section{Ultraproducts, axiomatisability and coherency}

For a monoid $S$ we denote by $L_{S}^{l}$ the first order language with equality, which has no constant or relation symbols and which has a unary function symbol $\lambda_{s}$ for each $s \in S$. We write $s x$ for $x \lambda_{s}$ and we regard left $S$-sets as $L_{S}^{l}$-structures in the obvious way. If $s, t \in S$ we denote by $\psi_{s, t}$ the sentence of $L_{S}^{l}$

$$
(\forall x)((s t) x=s(t x)) .
$$

Then an $L_{S}^{l}$-structure is a left $S$-set if and only if it is a model of $\Sigma_{S}^{l}=$ $\{(\forall x)(1 x=x)\} \cup\left\{\psi_{s, t}: s, t \in S\right\}$.

It seems natural to consider the conditions a monoid $S$ must satisfy in order that a given class of left $S$-sets be axiomatisable, that is, is precisely the class of models satisfying a given set of sentences of $L_{S}^{l}$. Here we find coherency notions appearing. Note that $\Sigma_{S}^{l}$ axiomatises the class of all left $S$-sets.

A major tool in investigating axiomatisability is Los's theorem.

TheORem 5.1 (Los, [6]). Let $L$ be a first order language and let $\mathscr{C}$ be a class of L-structures. If $\mathscr{C}$ is axiomatisable, then $\mathscr{B}$ is closed under ultraproducts.

In [12] we show that the class of existentially closed left $S$-sets, or the class of absolutely pure left $S$-sets, is axiomatsable if and only if $S$ is left 
coherent, and the class of weakly $f$-injective left $S$-sets is axiomatisable if and only if $S$ is weakly left coherent.

Theorem 3.1 of [11] states that the class of strongly flat left $S$-sets is axiomatisable if and only if for each $(a, b) \in S \times S, R(a, b)=\varnothing$ or is finitely generated (as a right $S$-subset of $S \times S)$ and $r(a, b)=\varnothing$ or is finitely generated. Moreover, this is equivalent to the property that every ultrapower of copies of $S$ is strongly flat as a left $S$-set. It is easy to see directly that such monoids must be weakly right coherent. On the other hand, this follows from Proposition 5.6 below.

In view of Los's theorem the problem arises of describing those monoids $S$ such that ultraproducts of certain left $S$-sets retain a given flatness property, and here again coherency plays a part. One can of course ask the corresponding question for products and it transpires that the answers are related. On the other hand we recall that in [4] a monoid $S$ is defined to be (weakly) right coherent if every non-empty product of (weakly) flat left $S$-sets is (weakly) flat.

Proposition 5.2. The following conditions are equivalent for a monoid $S$ :

(i) every product $S^{I} \quad(I \neq \varnothing)$ is strongly flat as left $S$-set;

(ii) every product $\prod\left\{A_{i}: i \in I\right\} \quad(I \neq \varnothing)$ of strongly flat left $S$-sets $A_{i}$, $i \in I$ is strongly flat;

(iii) for all $(a, b) \in S \times S, R(a, b)=\varnothing$ or is cyclic and $r(a, b)=\varnothing$ or is principal.

Proof. (i) $\Rightarrow$ (iii) Suppose that $a, b \in S$ and $R(a, b) \neq \varnothing$. Let $I$ index the set $R(a, b)$ and write $\left.R(a, b)=\left\{x_{i}, y_{i}\right): i \in I\right\}$. Let $\mathbf{x}, \mathbf{y}$ be the elements of $S^{I}$ whose $i$ 'th components are $x_{i}, y_{i}$ respectively. Then $a \mathbf{x}=b \mathbf{y}$ in $S^{I}$ and as $S^{I}$ is strongly flat by assumption, we have that $a u=b v, \mathbf{x}=u \mathbf{z}$ and $\mathbf{y}=v \mathbf{z}$ for some $u, v \in S$ and $\mathbf{z} \in S^{I}$. Thus $(u, v) \in R(a, b)$ so that $(u, v)=\left(x_{j}, y_{j}\right)$ for some $j \in I$. If $i \in I$ we have $\left(x_{i}, y_{i}\right)=(u, v) z_{i}=\left(x_{j}, y_{j}\right) z_{i}$ where $z_{i}$ is the $i$ 'th component of $\mathbf{z}$. This gives that $R(a, b)$ is cyclic. A similar argument applies to $r(a, b)$.

(iii) $\Rightarrow$ (ii) Let $P=\prod\left\{A_{i}: i \in I\right\}, I \neq \varnothing$, be a product of strongly flat left $S$-sets. Suppose that $a \mathbf{x}=b \mathbf{y}$ where $s, t \in S$ and $\mathbf{x}=\left(x_{i}\right), \mathbf{y}=$ $\left(y_{i}\right) \in P$. For each $i \in I, a x_{i}=b y_{i}$ and so as $A_{i}$ is flat there are elements $h_{i}, k_{i} \in S$ and $z_{i} \in A_{i}$ with $a h_{i}=b k_{i}, x_{i}=h_{i} z_{i}, y_{i}=k_{i} z_{i}$. Certainly $R(a, b) \neq \varnothing$ and so by assumption is cyclic, say $R(a, b)=(p, q) S$. Thus for each $i \in I,\left(h_{i}, k_{i}\right)=(p, q) r_{i}$ for some $r_{i} \in S$. We now have $a p=b q$ and $x_{i}=p r_{i} z_{i}, y_{i}=q r_{i} z_{i}$ for each $i \in I$. If $\mathbf{w}=\left(r_{i} z_{i}\right) \in P$, then $\mathbf{x}=p \mathbf{w}$ and $\mathbf{y}=q \mathbf{w}$. Together with a similar argument for equalities of the form $a \mathbf{x}=b \mathbf{x}$ this gives that $P$ is strongly flat. 
We now consider a further notion of coherency, which appears naturally when considering weakly flat ultraproducts of left $S$-sets and is moreover related to the work in [4].

A monoid $S$ is bounded weakly right coherent if given any $S$-homomorphism $\phi: \bigcup\left\{x_{i} S: 1 \leq i \leq n\right\} \rightarrow S$, then $\operatorname{ker} \phi=\rho(H)$ for some finite set $H$ such that the minimal lengths of the $\rho(H)$-sequences connecting pairs in $\operatorname{ker} \phi$ is bounded.

Given $\phi$ as above and a generating set $K$ of $\operatorname{ker} \phi$, then if $\left(x_{i} s, x_{j} t\right) \in$ $\operatorname{ker} \phi$ and

$$
x_{i} s=c_{1} t_{1}, d_{1} t_{1}=c_{2} t_{2}, \ldots, d_{l} t_{l}=x_{j} t
$$

is $\rho(K)$-sequence, we say that

$$
P=\left(c_{1}, d_{1}\right), \ldots,\left(c_{l}, d_{l}\right)
$$

is a path connecting $x_{i} s$ and $x_{j} t$.

If $S$ is such that given an $S$-homomorphism $\phi$ as above then $\operatorname{ker} \phi=\langle K\rangle$ for some $K$ with the property that for any $i, j \in\{1, \ldots, n\}$ then at most one path is needed to connect all elements of $\operatorname{ker} \phi$ of the form $\left(x_{i} s, x_{j} t\right)$, then $S$ is path weakly right coherent. Clearly, if $S$ is path weakly right coherent then $S$ is bounded weakly right coherent.

The proof of the following lemma is similar to that of [4, Proposition 2] and so is left to the reader.

Lemma 5.3. A monoid $S$ is path weakly right coherent if and only if for every finitely generated right ideal $I$ of $S$ and non-empty set $\Gamma$, the natural map $I \otimes S^{\Gamma} \rightarrow I^{\Gamma}$ is one-one.

The next result is now immediate from the definitions in [4].

Proposition 5.4. All left $S$-sets $S^{\Gamma}, \Gamma \neq \varnothing$, are weakly flat if and only if $S$ is path weakly right coherent

Thus if a monoid $S$ is weakly right coherent in the sense of [4] then it is weakly right coherent in our sense; this also follows indirectly from Corollary 3.3 and Theorem 4 of [4].

COROLLARY 5.5. Every left absolutely flat monoid is weakly right coherent.

We remark that Corollary 5.5 extends the result of Section 3 which states that every inverse monoid is weakly right coherent, for its is shown in [1] that inverse monoids are absolutely flat. Proposition 5.6 below compares 
with Proposition 5.4 in a manner analogous to that in which Theorem 3.1 of [11] compares with Proposition 5.2.

We adopt the notation $\mathbf{x}^{*}$ for the natural image in an ultrapower $S^{\gamma} / E$ of an element $\mathbf{x} \in S^{\gamma}$.

Proposition 5.6. Every ultrapower of $S$ is weakly flat as a left $S$-set if and only if $S$ is bounded weakly right coherent.

Proof. We suppose first that every ultrapower of $S$ is weakly flat in $S$-Ens. Let $\phi: \dot{\bigcup}\left\{x_{i} S: 1 \leq i \leq n\right\} \rightarrow S$ be an $S$-homomorphism and put $x_{i} \phi=c_{i}, 1 \leq i \leq n$, and $\rho=\operatorname{ker} \phi$. Assume that $\rho$ is not finitely generated.

Let $H=\left\{\left(x_{f(\beta)} u_{\beta}, x_{g(\beta)} v_{\beta}: \beta<\gamma\right\}\right.$ be a generating set for $\rho$ of minimum cardinality $\gamma$ such that if $\rho_{\alpha}=\left\langle\left\{\left(x_{f(\beta)} u_{\beta}, x_{g(\beta)} v_{\beta}\right): \beta<\alpha\right\rangle\right.$, then $\left(x_{f(\alpha)} u_{\alpha}, x_{g(\alpha)} v_{\alpha}\right) \notin \rho_{\alpha}$, for any $\alpha<\gamma$. Here $f, g$ are functions from $\gamma$ to $\{1, \ldots, n\}$. Let $D$ be a uniform ultrafilter on $\gamma$, that is, $D$ is an ultrafilter such that all sets in $D$ have cardinality $\gamma$. Put $\mathscr{U}=S^{\gamma} / D, \mathbf{u}=\left(u_{\beta}\right)$ and $\mathbf{v}=\left(v_{\beta}\right)$, so that $\mathbf{u}^{*}, \mathbf{v}^{*} \in \mathscr{U}$.

For each $i, j \in\{1, \ldots, n\}$ put $T_{i j}=\{\beta: f(\beta)=i, g(\beta)=j\}$. Then $\gamma=\bigcup\left\{T_{i j}: 1 \leq i, j \leq n\right\}$ and it follows that for some pair $(i, j), T_{i j} \in D$ : we fix for the moment this pair. For $\beta \in T_{i j}, c_{i} u_{\beta}=c_{j} v_{\beta}$ so that $c_{i} \mathbf{u}^{*}=c_{j} \mathbf{v}^{*}$ and it follows that $c_{i} \otimes \mathbf{u}^{*}=c_{j} \otimes \mathbf{v}^{*}$ in $S \otimes \mathscr{U}$. Then by assumption there is a scheme

$$
\begin{aligned}
c_{i}=a_{2} t_{1}, & \mathbf{u}^{*}=s_{1} \mathbf{b}_{1}^{*}, \\
a_{2} s_{2}=a_{3} t_{2}, & t_{1} \mathbf{b}_{1}^{*}=s_{2} \mathbf{b}_{2}^{*}, \\
\cdot & \cdot \\
a_{m} s_{m}=c_{j} t_{m}, & t_{m} \mathbf{b}_{m}^{*}=\mathbf{v}^{*},
\end{aligned}
$$

with $a_{2}, \ldots, a_{m} \in c_{i} S \cup c_{j} S$. It is easy to see that we may assume $a_{2}, \ldots$, $a_{m} \in\left\{c_{i}, c_{j}\right\}$. Pick a function $\{1, \ldots, m+1\} \rightarrow\{i, j\}$ written $k \rightarrow i_{k}$, such that $i_{1}=i, i_{m+1}=j$ and for $k \in\{2, \ldots, m\}, a_{k}=c_{i_{k}}$. Then for $k \in\{1, \ldots, m\}, x_{i_{k}} s_{k} \rho x_{i_{k+1}} t_{k}$. As $H$ generates $\rho$ and $\gamma$ is a limit ordinal, there is some $\alpha<\gamma$ with $x_{i_{k}} s_{k} \rho_{\alpha} x_{i_{k+1}} t_{k}$ for all $k \in\{1, \ldots, m\}$.

For $l \in\{1, \ldots, m\}$ we write $\mathbf{b}_{l}=\left(b_{l, \beta}\right)$ and define $V_{0}=\left\{\beta: u_{\beta}=\right.$ $\left.s_{1} b_{1, \beta}\right\}, V_{1}=\left\{\beta: t_{1} b_{1, \beta}=s_{2} b_{2, \beta}\right\}, \cdots, V_{m}=\left\{\beta: t_{m} b_{m, \beta}=v_{\beta}\right\}$. Thus $T=T_{i j} \cap V_{0} \cdots \cap V_{m}$ is in $D$ and as $D$ is uniform $T$ contains an element 
$\theta$ with $\alpha<\theta$. We now have that

$$
\begin{gathered}
x_{f(\theta)} u_{\theta}=x_{i} u_{\theta}=x_{i} s_{1} b_{1, \theta}=x_{i_{1}} s_{1} b_{1, \theta} \rho_{\alpha} x_{i_{2}} t_{1} b_{1, \theta}=x_{i_{2}} s_{2} b_{2, \theta} \rho_{\alpha} \cdots \rho_{\alpha} \\
x_{i_{m}} t_{m-1} b_{m-1, \theta}=x_{i_{m}} s_{m} b_{m, \theta} \rho_{\alpha} x_{j} t_{m} b_{m, \theta}=x_{j} v_{\theta}=x_{g(\theta)} v_{\theta}
\end{gathered}
$$

so that $x_{f(\theta)} u_{\theta} \rho_{\theta} x_{g(\theta)} v_{\theta}$, a contradiction. Hence $\operatorname{ker} \phi$ is finitely generated and $S$ is weakly right coherent.

To show that $S$ is bounded weakly right coherent, we consider $\phi$ as above and let $K$ be a finite set of generators of $\operatorname{ker} \phi$. We suppose by way of contradiction that for any $m \in N$ there is an element $\left(y_{m}, z_{m}\right)$ of $\operatorname{ker} \phi$ such that $y_{m}$ cannot be connected to $z_{m}$ be a $\rho(K)$-sequence of length equal to or less than $m$. Let $y_{m}=x_{f(m)} y_{m}^{\prime m}, z_{m}=x_{g(m)} z_{m}^{\prime}$ where $f, g: N \rightarrow$ $\{1, \ldots, n\}$ and $y_{m}^{\prime}, z_{m}^{\prime} \in S, m \in N$. Let $D$ be a non-principal ultrafilter over $N, \mathscr{U}=S^{N} / D$ and put $\mathbf{y}=\left(y_{m}^{\prime}\right), \mathbf{z}=\left(z_{m}^{\prime}\right)$.

For each $i, j \in\{1, \ldots, n\}$ let $T_{i j}=\{m: f(m)=i, g(m)=j\}$ so that $N=\bigcup\left\{T_{i j}: 1 \leq i, j \leq n\right\}$ and we may choose and fix some $T_{i j} \in D$. If $m \in T_{i j}$ then $\left(y_{m}, z_{m}\right)=\left(x_{i} y_{m}^{\prime}, x_{j} z_{m}^{\prime}\right)$ and since $\left(y_{m}, z_{m}\right) \in \operatorname{ker} \phi$, $c_{i} y_{m}^{\prime}=c_{j} z_{m}^{\prime}$, giving that $c_{i} \mathbf{y}^{*}=c_{j} \mathbf{z}^{*}$ and $c_{i} \otimes \mathbf{y}^{*}=c_{j} \otimes \mathbf{z}^{*}$ in $S \otimes \mathscr{U}$. Again we have a typical scheme.

$$
\begin{aligned}
& \mathbf{y}^{*}=s_{1} \mathbf{b}_{1}^{*}, \\
& c_{i} s_{1}=a_{2} t_{1}, \quad t_{1} \mathbf{b}_{1}^{*}=s_{2} \mathbf{b}_{2}^{*} \text {, } \\
& a_{2} s_{2}=a_{3} t_{2} \text {, } \\
& a_{m} s_{m}=c_{j} t_{m}, \quad t_{m} \mathbf{b}_{m}^{*}=\mathbf{z}^{*},
\end{aligned}
$$

where we take $a_{2}, \ldots, a_{m} \in\left\{c_{i}, c_{j}\right\}$; we pick a function $\{1, \ldots, m+1\} \rightarrow$ $\{i, j\}$, written $k \mapsto i_{k}$, as in the first part of the proof. Then for $k \in$ $\{1, \ldots, m\}, x_{i_{k}} s_{k} \rho x_{i_{k+1}} t_{k}$ and amongst the $\rho(K)$-sequences joining $x_{i_{k}} s_{k}$ to $x_{i_{k+1}} t_{k}$ there must be one of shortest length $p_{k}$. Choose and fix such a sequence for each $k \in\{1, \ldots, m\}$.

For $l \in\{1, \ldots, m\}$ put $\mathbf{b}_{l}=\left(b_{l, r}\right)$ and let $V_{0}=\left\{r: y_{r}^{\prime}=s_{1} b_{1, r}\right\}$, $V_{1}=\left\{r: t_{1} b_{1, r}=s_{2} b_{2, r}\right\}, \ldots, V_{m}=\left\{r: t_{m} b_{m, r}=z_{m}^{\prime}\right\}$. Then $T=T_{i j} \cap V_{0} \cap$ $\cdots \cap V_{m} \in D$ and so there is an element $q$ in $T$ with $q>p_{1}+\cdots+p_{m}$.

By assumption, $\left(y_{q}, z_{q}\right)=\left(x_{i} y_{q}^{\prime}, x_{j} z_{q}^{\prime}\right)$ is such that $y_{q}$ cannot be related to $z_{q}$ by a $\rho(K)$-sequence of length equal to or less than $q$. But $x_{i} y_{q}^{\prime}=x_{i_{1}} s_{1} b_{1, q} \rho x_{i_{2}} t_{1} b_{1, q}=x_{i_{2}} s_{2} b_{2, q} \rho \cdots \rho x_{i_{m+1}} t_{m} b_{m, q}=x_{j} z_{q}^{\prime}$, which gives a $\rho(K)$-sequence of length $p_{1}+\cdots+p_{k}$, a contradiction. It follows that $S$ is bounded weakly right coherent. 
Conversely, we assume that $S$ is bounded weakly right coherent and consider an ultrapower $\mathscr{U}=S^{\gamma} / D$. In view of Exercises 4.1.6 and 4.1.21 of [6] we assume that $\gamma$ is infinite and $D$ is non-principal. We suppose that $a \otimes \mathbf{x}^{*}=b \otimes \mathbf{y}^{*}$ in $S \otimes \mathscr{U}$ so that in $\mathscr{U}$ we have $a \mathbf{x}^{*}=b \mathbf{y}^{*}$. Define $\phi: w S \dot{\cup} z S \rightarrow S$ by $w \phi=a$ and $z \phi=b$. Then there is a finite set $H \subseteq \operatorname{ker} \phi$ such that $\operatorname{ker} \phi=\rho(H)$ and the minimal lengths of $\rho(H)$-sequences connecting the coordinates of elements of $\operatorname{ker} \phi$ is bounded. Writing $\mathbf{x}=\left(x_{i}\right)$ and $\mathbf{y}=\left(y_{i}\right)$ we let $T=\left\{i \in \gamma: a x_{i}=b y_{i}\right\}$, so that $T \in D$. Let $P_{1}, \ldots, P_{r}$ label the paths of $\rho(H)$-sequences needed to connected elements of $\operatorname{ker} \phi$. For each $i \in T, w x_{i} \rho(H) z y_{i}$ and so we choose and fix a path $P_{f(i)}$ such that $w x_{i}$ is connected to $z y_{i}$ via this path.

Define $T_{k}=\{i \in T: f(i)=k\}, k \in\{1, \ldots, r\}$, so that $T=T_{1} \cup \cdots \cup T_{r}$ and for some $k \in\{1, \ldots, r\}, T_{k} \in D$. Let us fix this $k$. Then for any $j \in T_{k}$ there is a path

$$
w x_{j}=c_{1} t_{1 j}, d_{1} t_{1 j}=c_{2} t_{2 j}, \ldots, d_{l} t_{l j}=z y_{j}
$$

where $\left(c_{i}, d_{i}\right) \in H \cup H^{-1}, 1 \leq i \leq l$. For $i \in\{1, \ldots, l\}$ let $\mathbf{t}_{l} \in S^{\gamma}$ have $j$ 'th coordinate $t_{l j}$ for $j \in T_{k}$ (and be arbitrary otherwise). Write $\left(c_{i}, d_{i}\right)$ as $\left(x_{g(i)} u_{i}, x_{h(i)} v_{i}\right)$. This gives that

$$
a \otimes \mathbf{x}^{*}=a \otimes u_{1} \mathbf{t}_{1}^{*}=a u_{1} \otimes \mathbf{t}_{1}^{*}=\cdots=b v_{l} \otimes \mathbf{t}_{l}^{*}=b \otimes v_{l} \mathbf{t}_{l}^{*}=b \otimes \mathbf{y}^{*}
$$

and so $a \otimes \mathbf{x}^{*}=b \otimes \mathbf{y}^{*}$ in $a S \cup b S \otimes \mathscr{U}$. Thus $\mathscr{U}$ is weakly flat.

It is a consequence of the proof of Proposition 5.6 that if $S$ is bounded weakly right coherent, then given an $S$-homomorphism $\phi: x_{1} S \cup \cdots \cup x_{n} S \rightarrow$ $S$, it is true that for any finite set $H$ of generators of $\operatorname{ker} \phi$, the minimal lengths of the $\rho(H)$-sequences needed to connect pairs in $\operatorname{ker} \phi$ is bounded.

COROLlaRy 5.7. If the weakly flat (flat, strongly flat) left $S$-sets are an axiomatisable class, then $S$ is bounded weakly right coherent.

FROCF. This follows from Theorem 5.1 and Proposition 5.6.

\section{References}

[1] S. Bulman-Fleming and K. McDowell, 'Absolutely flat semigroups', Pacific J. Math. 107 (1983), 319-333.

[2] S. Bulman-Fleming and K. McDowell, 'Left absolutely flat generalized inverse semigroups', Proc. Amer. Math. Soc. 94 (1985), 553-561. 
[3] S. Bulman-Fleming and K. McDowell, 'Monoids over which all weakly flat acts are flat', Proc. Edinburgh Math. Soc. 33 (1990), 287-298.

[4] S. Bulman-Fleming and K. McDowell, 'Coherent monoids', in: Lattices, Semigroups and Universal Algebra, ed. J. Almeida et al. (Plenum Press, N.Y. 1990).

[5] S. U. Chase, 'Direct product of modules', Trans. Amer. Math. Soc. 97 (1960), 457-473.

[6] C. C. Chang and H. J. Keisler, Model Theory (North-Holland, Amsterdam, New-York, Oxford 1973).

[7] A. H. Clifford and G. B. Preston, The algebraic theory of semigroups, Math. Surveys 7 (American Mathematical Society, 1961).

[8] C. Faith, Rings, Modules and Categories I, (Springer-Verlag, Berlin, 1973).

[9] J. B. Fountain, 'A class of right PP monoids', Quart. J. Math. Oxford 28 (1977), 285300.

[10] J. B. Fountain, 'Abundant Semigroups', Proc. London Math. Soc. 44 (1982), 103-129.

[11] V. A. R. Gould, 'Axiomatisability problems for $S$-systems', J. London Math. Soc. 35 (1987), 193-201.

[12] V. A. R. Gould, 'Model companions of S-systems', Quart. J. Math. Oxford 38 (1987), 189-211.

[13] J. M. Howie, An introduction to semigroup theory (Academic Press, 1976).

[14] M. Kilp, 'Commutative monoids all of whose principal ideals are projective', Semigroup Forum 6 (1973), 334-339.

[15] U. Knauer, 'Projectivity of acts and Morita equivalence of monoids', Semigroup Forum 3 (1972), 359-370.

[16] U. Knauer and M. Petrich, 'Characterization of monoids by torsion-free, flat, projective and free acts', Archiv der Math. 36 (1981), 289-294.

[17] J. Lambek, Lectures on Rings and Modules, (Blaisdell Publishing Co., 1976).

[18] P. Normak, 'On noetherian and finitely presented polygons', Uc. Zap. Tartu Gos. Univ. 431 (1977), 37-46.

[19] L. Redei, Theorie der endlich erzeugbaren kommutativen Halbgruppen, Hamburger mathematische Einzelschriften, Heft 41 (Physica-Verlag, Warzburg 1963).

[20] J. Rotman, An Introduction to Homological Algebra (Academic Press, New-York, 1979).

[21] B. Stenstrom, 'Flatness and localisation over monoids', Math. Nach. 48 (1971), 315-334.

[22] W. H. Wheeler, 'Model companions and definability in existentially complete structures', Israel J. Math. 25 (1976), 305-330.

\section{University of York \\ Heslington, York YO1 5DD \\ United Kingdom}

